\title{
Comparative Study of Risk Factors for Opaque Bubble Layer Formation in Femtosecond-Laser Assisted LASIK
}

\author{
AHMED S. ABDEL WANES, M.Sc.*; ABDELRAHMAN G. SALMAN, M.D.**; AZZA M.A. SAID, M.D.** and \\ BASSEM F.A. RIAD, M.D.**
}

The Department of Ophthalmology, Faculty of Medicine, Zagazig* and Ain Shams** Universities**

\begin{abstract}
Background: Refractive surgery had undergone a remarkable evolution during the last 25 years. The introduction of excimer laser in practice of refractive surgery was an exciting innovation.

Aim of Study: To determine risk factors responsible for opaque bubble layer formation in femtosecond-laser assisted LASIK.

Patients and Methods: This retrospective comparative study was conducted on patients who were eligible for FSLassisted LASIK at El-Watany Eye Hospital in the period from January 2015 to January 2017. Two hundred eyes were included in the study divided into 100 had OBL developed during surgeries and 100 did not develop OBL.

Results: The results of the present study revealed a statistically significant difference between both groups regarding preoperative central corneal thickness, corneal canal length offset and steep keratometry being higher in OBL formation group. Regarding regression analysis central corneal thickness and corneal astigmatism were statistically significant independent predictor of OBL formation.

Conclusion: OBL is one of FSL complications. Increased corneal thickness, steep keratometry and increased corneal canal length offset and were risk factors of OBL formation during surgeries. Regarding regression analysis; central corneal thickness and corneal astigmatism were statistically significant independent predictor of OBL formation.
\end{abstract}

Key Words: Opaque bubble layer - Femtosecond-LaserLASIK.

\section{Introduction}

LASER-ASSISTED in situ keratomileusis (LASIK) is a widely accepted method for correcting the refractive error [1]. In recent years, the use of bladeless LASIK surgery utilizing a Femtosecond Laser (FSL) (named for its ultrashort pulses, with duration of few femtoseconds) for lamellar flap

Correspondence to: Dr. Ahmed S. Abdel Wanes, E-Mail: ahmad wanees@yahoo.com creation, as an alternative option to the mechanical microkeratome (MK) [2].

A second laser involved in the procedure, the excimer provides the ablation and has also evolved significantly over the course of the past 10 years. Excimer lasers for refractive surgery can provide customized ablation, including aspheric ablation profiles, wavefront-guided, or topography-guided treatments [3].

Femtosecond-Laser (FSL) becomes one of the most important advances in refractive surgery as it increases the predictability, precision and accuracy of LASIK flap creation. Also, it allows for flap customization (thickness, diameter, side cut angle) and is better for thin cornea high spherical ametropia and high astigmatism [4].

On the other side, this advanced procedure presents specific kind of complications; one of them is Opaque Bubble Layer (OBL) which produced by gas bubbles that accumulate in the superficial layers of the stromal bed during FSL [5]

FSL pulses are focused in the corneal tissue where ionization occur. Plasma expansion in the tissue creates cavitation bubbles causing separation between stromal lamellae. OBL is the accumulation of gas bubbles temporarily detained in the intrastromal interface, creating transient opacity [1].

Previous studies have described two different types of OBLs. "Soft", "diffuse", or "delayed" OBLs have a more transparent appearance and occur later, after completion of laser dissection in a particular area. "Hard", "advancing", or "early" OBLs appear earlier and have a denser appearance $[1,6]$. 

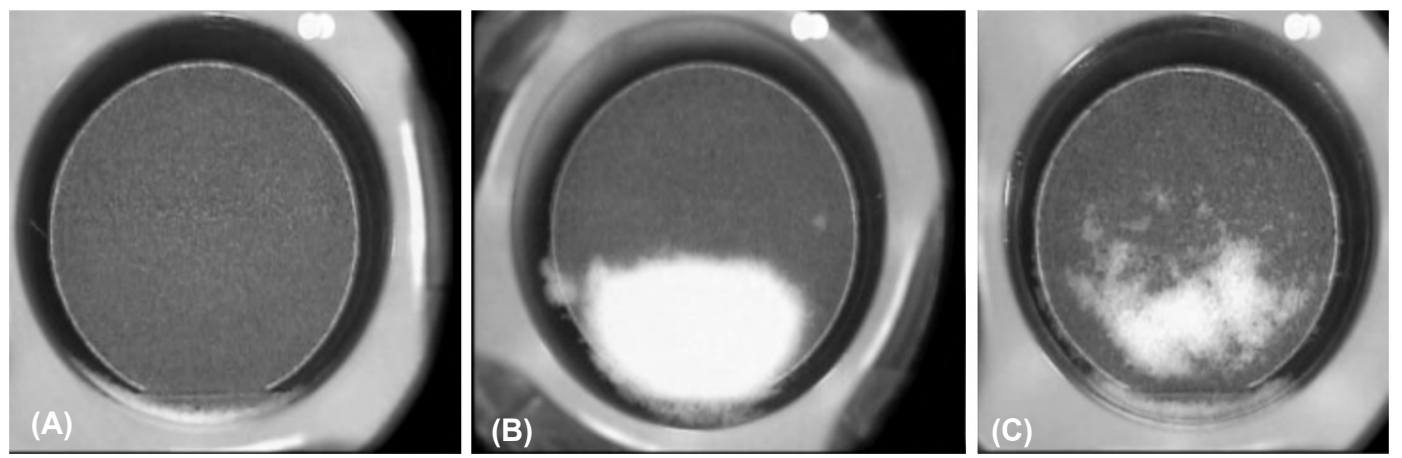

Fig. (1): Representative photographs of no OBL (A), a hard OBL (B), and a soft OBL (C) (Liu et al., 2014).

The trapped gas may create difficulty in lifting the LASIK flap, as the gas has not remained in one dissection plane within the cornea. Depending on the location and extent of the OBL, it may interfere with pupil tracking during excimer laser ablation if it obscures all or part of the pupil, and it can prevent iris-registered tracking during ablation if it obstructs tracking software from identifying patient-specific iris characteristics. Furthermore, an OBL may limit a patient's ability to fixate properly during ablation [4].

Several studies reported the incidence rate, risk factors and clinical relevance of OBL. Courtin et al., 2015 found that $48 \%$ of eyes had FSL assisted LASIK had OBL owing to thicker corneas and increased corneal resistance factor and corneal hysteresis.

This was in agreement with Liu et al., 2014 who reported an incidence rate of $52.5 \%$ and Kasierman et al., 2008 who stated that thicker corneas and smaller flaps were associated with a more OBLs with an incidence rate of $56.4 \%$.

In fact, gas bubbles produced by a FSL have been shown to travel the pathway of least resistance, and under high pressure due to high vacuum, corneal compression and corneal rigidity can reactively produce a counterbalance force to oppose the applanation pressure. Thicker corneas can provide greater rigidity and produce more resistance, thereby restricting the clearance of the cavitation bubbles and increasing the occurrence of OBLs [7].

An oval-shaped flap with a larger hinge angle tended to result in less OBL formation in femtoLASIK. Also, the wider canal settings along with customized denser spot application significantly reduce the risk of OBL [6].

\section{Patients and Methods}

This retrospective comparative study was conducted on patients who were eligible for FSL- assisted LASIK at El-Watany Eye Hospital in the period from January 2015 to January 2017.

Patient selection:

- Inclusion criteria:

- Age: 18-40 years old.

- Either myope or hyperope with or without astigmatism.

- Candidate for laser refractive procedure.

- Exclusion criteria:

- Previous refractive procedures.

- Previous corneal pathologies (e.g. opacities, ulcers, ... ).

- Previous corneal surgeries (e.g. ptergyium, PKP, ...).

- Local or systemic diseases or medications.

- Eyes at risk for developing post-refractive corneal ectasia, such as keratoconus and keratoconus suspect.

Those patients were divided into two groups:

- Study group: 100 eyes had OBL formation during surgery.

- Control group: 100 eyes didn't develop OBL during surgery.

Ethical considerations: All procedures including possible side effects were explained to the patients and they provided us with an informed written consent. This study was adhered to the tenets of the declaration of Helsinki.

Study tools: Medical records of patients who were included in the study were reviewed and the following data were collected:

- Best Corrected Visual Acuity (BCVA) using automated chart projector Snellen's chart with conversion of the values to logarithm of minimum angle of resolution (Log. MAR) for statistical analysis. 
- Spherical Equivalent (SE) powers.

- Intra Ocular Pressure (IOP) measurements using Goldman's applanation tonometer attached to the slit-lamp.

- Posterior segment examination results using indirect ophthalmoscope (Keeler Ltd. Windsor, UK) (using handheld +20D lens) and slit lamp biomicroscopy using handheld +90D lens (Volk Optical Mentor, $\mathrm{OH}$ ).

- Topography examination: It's the cornerstone for evaluation of cases that fit for the surgery or not, for this reason, all topography examinations have to be performed with great care. Only well-trained personnel could perform, validate and export examinations.

- Corneal tomography results including Keratometric readings including flat keratometric reading (K1), steep keratometric reading (k2) and central corneal thickness (CCT) measurements using (Pentcam R HR type 70900-OCCULUSoptikgerate Gmbh).

The following during the tomography examination with Pentcam should be considered:

- The eye to be examined shouldn't have applanation tonometry or contact pachymetry during 12 hours prior to the tomography examination.

- Contact lens wearer must discontinue the wear for at least 7 days prior to the pre-operative evaluation.

- A proper tear film is essential for good image quality.

- The patient was instructed about what she/he has to do, what she/he and should avoid and what she/he will notice during examination. Tomography examination as well as image and data validation were performed. Examination procedure steps and validation checkpoints shall include, but didn't be limited to the following:

- Quality specification was confirmed.

- Good centration of patient head.

- The captured image (shadow of nose and eyelid) was checked shows the printout of the four refractive maps of corneal tomography.

- FSL was done using (FS200, wavelight, Germany SR/1025-1-380).

Operation steps (surgeries were performed by one of the authors):

- The data on the laser machine matched with the patient and treated eye. The eye showed on the laser LCD screen.
- Other data entery included patient refraction pachymetry, $\mathrm{k}$ readings, flap hinge angle, canal length, flap thickness and diameter were recorded.

- Pupil size for treatment was within $2 \mathrm{~mm}$ of the size during the tomography examination. Medications likely to dilate the pupil were administered with careful supervision prior to surgery.

- Laser calibration included bed cut (spot separation, line separation, pulse energy) and side cut (spot separation, line separation, pulse energy) was done.

- The procedure was performed under topical anesthesia.

- Alignment mark using (gentian violet or methylene blue).

- Pneumatic suction ring was applied to the eye (while applying the suction ring, the cornea must be dry).

- Suction ring was applied for good centration of the cornea before docking then docking system flatten the corneal surface artificially by applying equal pressure over the corneal surface.

- Flap creation using FSL.

- OBL formed or not introperatively was documented.

- Data of the present study collected from treatment report.

Serious problems of OBL did not encountered. However, after flap creation complicated with OBL, we found subjectively that flaps were more difficult to lift. If OBL was excessive and flap lifting was difficult, surgeon could wait for few minutes until the trapped gas dissolved or by pressing smoothly near this emphysematous like pockets, the OBL disappeared.

In some cases, OBL limited patient's ability to fixate on target so it may interfere with pupil tracker during excimer laser part.

\section{Statistical analysis:}

The collected data were analyzed by computer using Statistical Package of Social Services (IBM SPSS) version 24 (SPSS Inc., 2017 South Wacker Drive, Chicago, USA), Data were represented in tables and graphs, continuous quantitative variables e.g. age were expressed as the mean \pm SD and median (Interquartile Range; IQR), and categorical qualitative variables were expressed as absolute frequencies (number) \& relative frequencies (percentage). 
The arithmetic mean as an average describing the central tendency of observation.

The Standard Deviation (SD) as a measure of dispersion of the results around the mean.

The arithmetic median as a middle value when the data are arranged serially.

Range lies from the lowest to the highest observation.

- Suitable statistical tests of significance were used after checked for normality.

Mann-Whitney U (MW test) was used for comparing numerical variables between 2 groups, it is the non-parametric equivalent of $t$ test, was used if the data cannot be assumed to have a normal distribution.

Chi-square test $\left(\chi^{2}\right)$ was used for comparing numerical variables between the two groups; it was used if the data cannot be assumed to have a normal distribution and/or Fisher exact test only when the expected count in any cell found less than 5 .

Spearman's correlation coefficient was used to assess the correlation between two variables in the study group.

Logistic regression analysis was used for modeling and analyzing several variables with focusing on relationship between a dependent variable and one or more independent variables or predictors.

The results were considered statistically significant when the significant probability was equal or less than $0.05(p \leq 0.05)$.

$p$-value $\leq 0.001$ was considered highly statistically significant (HS), and $p$-value $>0.05$ was considered statistically insignificant (NS).

\section{Results}

The present study was conducted on 200 eyes among 101 patients to study risk factors of OBL occurrence after FSL. 100 eyes had OBL and 100 eyes had no OBL retrospectively.

\section{I- Demographic data:}

1-Age: The median age of the study group was 30 years (range: 20-40; IQR: 11) (Table 1).

2- Sex: Sixty-four patients $(63.4 \%)$ of the studied sample were females, and 37 patients $(36.6 \%)$ of them were males (Table 2).
Table (1): Age of the studied patients.

\begin{tabular}{|c|c|c|}
\hline Demographic data & \multicolumn{2}{|c|}{ Studied patients $(\mathrm{N}=101)$} \\
\hline Age (years): & \multirow{3}{*}{\multicolumn{2}{|c|}{$\begin{array}{l}30 \text { years, } 11 \text { years } \\
20-40\end{array}$}} \\
\hline Median, IQR & & \\
\hline Range & & \\
\hline \multicolumn{3}{|c|}{ Table (2): Sex distribution among the studied patients. } \\
\hline \multirow{2}{*}{ Demographic data } & \multicolumn{2}{|c|}{ Studied patients $(\mathrm{N}=101)$} \\
\hline & No. & $\%$ \\
\hline \multicolumn{3}{|l|}{ Sex: } \\
\hline Male & 37 & 36.6 \\
\hline Female & 64 & 63.4 \\
\hline
\end{tabular}

II- Pre-operative assessment (Table 3): One hundred ninety-four eyes $(97 \%)$ were myopic and 6 eyes (3\%) were hyperopic.

1-Spherical equivalent (SE): The median SE was -3.25 diopters (ranged from -10.75 to +3.25 ; IQR: +2.75).

2- Corneal astigmatism: The median astigmatism was -1.05 diopters (ranged from -0.1 to -4.9 ; IQR: 1.08).

3- Corneal keratometry: The mean flat keratometry (k1) was 42.95 diopters \pm 1.54 SD (range: 38.8 46.9) while the mean steep keratometry (k2) was 44.22 diopters \pm 1.69 SD (range: $39.5-49.2$ ).

4- Central corneal thickness ( $\mathbf{m})$ :The median central CCT was 545 mange: 484-600; IQR: 46).

Table (3): Pre-operative corneal parameters among the studied eyes.

\begin{tabular}{llll}
\hline \multirow{2}{*}{ Corneal parameters } & \multicolumn{3}{c}{ The studied eyes $(\mathrm{n}=200)$} \\
\cline { 2 - 4 } & Median & IQR & \multicolumn{1}{c}{ Range } \\
\hline Spherical equivalent (diopters) & -3.25 & +2.75 & -10.75 to+3.25 \\
Astigmatism (diopters) & -1.05 & 1.08 & -0.1 to-4.9 \\
Flat keratometry (k1) (diopters) & $42.95 *$ & $1.54 * *$ & $38.8-46.9$ \\
Steep keratometry (k2) (diopters) & $44.22^{*}$ & $1.69 * *$ & $39.5-49.2$ \\
Central corneal thickness $(\mu \mathrm{m})$ & 545.00 & 46 & $484-600$ \\
\hline *: Mean. $\quad * *:$ SD. & & & \\
\hline
\end{tabular}

III- Intraoperative assessment: For FSL flap creation, the set parameters were flap diameter, flap thickness, and flap bed cut, side cut, and hinge were WaveLight FS200 laser default settings as shown in (Tables 4A,4B).

1-Flap diameter: The mean corneal flap diameter was $8.76 \mathrm{~mm} \pm 0.07 \mathrm{SD}$, ranged from 8.6 to 9.0 $\mathrm{mm}$. Corneal flap diameter was $8.7 \mathrm{~mm}$ in $43.5 \%$ of the studied eyes and it was $\geq 8.8 \mathrm{~mm}$ among $56.5 \%$ of the studied eyes.

2- Flap thickness: The mean flap thickness was 117.28um $\pm 5.22 \mathrm{SD}$, ranged from 100 to 200 
Corneal flap thickness was $\leq 115(\mathrm{~m}) 25.5 \%$ of the studied eyes and it was 120 ( m) among $74.5 \%$ of the studied eyes.

3-Laser parameters: Bed cut spot separations and line separations ranged from 7.0 to $8.0 \mathrm{~m}$ vhile spot separations and line separations in side cut ranged from 4.5 to $5.0 \mathrm{rm}$ and pulse energy ranged from 0.67 to 0.84 ind cut and side cut, flap hinge angle ranged from 49 to $51^{\circ}$ and canal length offset ranged from 0.10 to $1.00 \mathrm{~mm}$.

Table (4A): Femtosecond laser flap creation settings.

\begin{tabular}{llll}
\hline & \multicolumn{3}{c}{ Measurement } \\
\cline { 2 - 4 } Setting & Mean & SD & \multicolumn{1}{c}{ Range } \\
\hline $\begin{array}{l}\text { Corneal flap: } \\
\quad \text { Diameter }(\mathrm{mm})\end{array}$ & 8.76 & 0.07 & 8.60 to 9.0 \\
$\quad$ Thickness $(\mu \mathrm{m})$ & 117.28 & 5.22 & 100.0 to 120.0 \\
& & & \\
Bed cut: & 7.45 & 0.269 & 7.0 to 8.0 \\
$\quad$ Line separations $(\mu \mathrm{m})$ & 0.791 & 0.025 & 0.67 to 0.84 \\
$\quad$ Pulse energy $(\mu \mathrm{j})$ & & & \\
Side cut: & & & \\
$\quad$ Spot separations $(\mu \mathrm{m})$ & 4.90 & 0.20 & 4.5 to 5.0 \\
$\quad$ Line separations $(\mu \mathrm{m})$ & 2.88 & 0.51 & 0.3 to 5.0 \\
$\quad$ Pulse energy $(\mu \mathrm{j})$ & 0.791 & 0.025 & 0.67 to 0.84 \\
Flap hinge angle & 49.96 & 0.24 & 49 to $51^{\circ}$ \\
$\quad$ Canal length offset $(\mathrm{mm})$ & 0.545 & 0.28 & 0.10 to 1.00 \\
\hline
\end{tabular}

Table (4B): Corneal flap diameter and thickness among the studied patients.

\begin{tabular}{llc}
\hline & \multicolumn{2}{l}{ Studied eyes $(\mathrm{N}=200)$} \\
\cline { 2 - 3 } Corneal flap & No. & $\%$ \\
\hline Corneal flap diameter $(\mathrm{mm}):$ & & \\
$8.7(\mathrm{~mm})$ & 87 & 43.5 \\
$\geq 8.8(\mathrm{~mm})$ & 113 & 56.5 \\
Corneal flap thickness $(\boldsymbol{m})$ & & \\
$\leq 115(\mu \mathrm{m})$ & 51 & 25.5 \\
$120(\mu \mathrm{m})$ & 149 & 74.5 \\
\hline
\end{tabular}

4- Opaque Bubble Layer (OBL) formation: Among the studied 101 patients, OBL occurs bilaterally in 58 eyes among 29 patients, while non-OBL occurs bilaterally in 58 eyes among 29 patients, and OBL occurs unilaterally among 41 patients, the remaining 2 patients, only one eye was studied in each one, OBL occurs in one patient and non-OBL occurs in the other patient. Being a retroactive study, it is difficult to differentiate the type o OBL formed either soft or hard.

\section{A- Demographic data:}

- Age (years): The median age of patients developed OBL and those had no OBL formed were; 29.5 years and 30.5 years respectively. No statistically significant difference was found as regards age between both groups (Table 5).
- Sex: Regarding sex distribution, there was no statistical difference between both groups regarding sex, where 27 patients $(37.5 \%)$ of the studied OBL group were males and 10 patients $(34.4 \%)$ of the studied non-OBL patients were male (Table $5)$.

Table (5): Comparison between OBL and non-OBL regarding age and sex of the studied patients.

\begin{tabular}{|c|c|c|c|c|c|c|}
\hline \multirow{2}{*}{ Variable } & \multicolumn{2}{|c|}{ OBL } & \multicolumn{2}{|c|}{ Non-OBL } & \multirow{2}{*}{$\frac{x^{2}}{\text { Z-value/ }}$} & \multirow{2}{*}{$p$-value } \\
\hline & No. & $\%$ & No. & $\%$ & & \\
\hline Age (years): & \multirow{2}{*}{\multicolumn{2}{|c|}{$\begin{array}{l}29.5,10.0 \\
(20-40)\end{array}$}} & \multirow{2}{*}{\multicolumn{2}{|c|}{$\begin{array}{l}30.5,15.25 \\
(20-40)\end{array}$}} & & \\
\hline $\begin{array}{l}\text { Median, IQR } \\
\text { Range }\end{array}$ & & & & & -1.07 & 0.28 \\
\hline \multicolumn{7}{|l|}{ Sex: } \\
\hline Male & 27 & 37.5 & 10 & 34.4 & 0.081 & 0.775 \\
\hline Female & 45 & 63.5 & 19 & 65.6 & & \\
\hline
\end{tabular}

B-Pre-operative corneal parameters in relation to Opaque Bubble Layer formation:

- Spherical equivalent (SE): Regarding preoperative SE in relation to OBL formation among the studied 200 eyes, there was no statistically significant difference between both groups ( $p$ value was 0.64 )

On distribution of SE, there was no statistically significant difference between OBL and non-OBL ( $p$-value was 0.91$)$ where SE was more than -2.25 in 23 eyes $(23 \%)$ of OBL group versus 26 eyes $(26.0 \%)$ in non-OBL eyes (Table 6).

Table (6): Pre-operative spherical equivalent in relation to OBL formation among the studied eyes.

\begin{tabular}{|c|c|c|c|c|c|c|}
\hline \multirow[t]{2}{*}{ SE (diopters) } & \multicolumn{2}{|c|}{$\begin{array}{c}\text { OBL } \\
(\mathrm{N}=100) \text { eyes }\end{array}$} & \multicolumn{2}{|c|}{$\begin{array}{c}\text { Non-OBL } \\
(\mathrm{N}=100) \text { eyes }\end{array}$} & \multirow{2}{*}{$\begin{array}{c}\mathrm{Z}- \\
\text { value/ } \\
\chi^{2}\end{array}$} & \multirow{2}{*}{$\begin{array}{c}p- \\
\text { value }\end{array}$} \\
\hline & No. & $\%$ & No. & $\%$ & & \\
\hline \multicolumn{7}{|l|}{$S E$ : } \\
\hline $\begin{array}{l}\text { Median, IQR } \\
\text { Range }\end{array}$ & \multicolumn{2}{|c|}{$\begin{array}{l}-3.25,2.75 \\
(-8.75 \text { to }+3.25)\end{array}$} & \multicolumn{2}{|c|}{$\begin{array}{l}-3.25,3.25 \\
(-10.75 \text { to }+3)\end{array}$} & -0.463 & 0.64 \\
\hline \multicolumn{7}{|l|}{$S E$ : } \\
\hline $\begin{array}{l}\text { Lowest thru }-5.0 \\
-5 \text { thru }-3.25 \\
-3.25 \text { thru }-2.25 \\
-2.25 \text { thru highest }\end{array}$ & $\begin{array}{l}26 \\
27 \\
24 \\
23\end{array}$ & $\begin{array}{l}26.0 \\
27.0 \\
24.0 \\
23.0\end{array}$ & $\begin{array}{l}28 \\
24 \\
22 \\
26\end{array}$ & $\begin{array}{l}28.0 \\
24.0 \\
22.0 \\
26.0\end{array}$ & 0.521 & 0.91 \\
\hline
\end{tabular}

- Corneal astigmatism: Regarding preoperative astigmatism among the studied 200 eyes, there was no statistically significant difference between both groups ( $p$-value was 0.60) (Table 7).

Table (7): Mean of astigmatism in relation to OBL formation among the studied eyes.

\begin{tabular}{lllrl}
\hline $\begin{array}{l}\text { Astigmatism } \\
\text { (diopters })\end{array}$ & $\begin{array}{c}\text { OBL } \\
(\mathrm{N}=100) \text { eyes }\end{array}$ & $\begin{array}{c}\text { Non-OBL } \\
(\mathrm{N}=100) \text { eyes }\end{array}$ & $\begin{array}{c}\mathrm{Z}- \\
\text { value }\end{array}$ & $\begin{array}{c}p \text { - } \\
\text { value }\end{array}$ \\
\hline Median & -1.0 & -1.1 & -0.52 & 0.60 \\
IQR & 1.1 & 1.0 & & \\
Range & $(-0.1$ to -4.4$)$ & $(-0.1$ to -4.9$)$ & & \\
\hline
\end{tabular}


- Refraction: In OBL group, 98 eyes $(98.0 \%)$ were myopic, and only 2 eyes ( $2 \%$ ) were hypermetropia while in non-OBL 96 eyes $(96.0 \%)$ were myopic, and 4 eyes (4\%) were hypermetropia with no statistically significant difference between OBL and non-OBL regarding refraction (Table 8).

Table (8): Refraction among the studied group.

\begin{tabular}{|c|c|c|c|c|c|c|}
\hline \multirow[t]{2}{*}{ Refraction } & \multicolumn{2}{|c|}{$\begin{array}{c}\text { OBL } \\
(\mathrm{N}=100)\end{array}$} & \multicolumn{2}{|c|}{$\begin{array}{c}\text { Non-OBL } \\
(\mathrm{N}=100)\end{array}$} & \multirow{2}{*}{$\chi^{2}$} & \multirow{2}{*}{$\begin{array}{c}p- \\
\text { value }\end{array}$} \\
\hline & No. & $\%$ & No. & $\%$ & & \\
\hline Myopia & 98 & 98.0 & 96 & 96.0 & 0.687 & 0.704 \\
\hline Hypermetropia & 2 & 2.0 & 4 & 4.0 & & \\
\hline
\end{tabular}

- Flat keratometry ( $k 1)$ : Regarding pre-operative flat keratometric reading (k 1) among the studied 200 eyes, there was no statistically significant difference between both groups ( $p$-value was 0.17) (Table 9).

Regarding the distribution of flat keratometric reading $(\mathrm{K} 1)$, there was no statistically significant difference between OBL and non-OBL ( $p$-value was 0.15 ) where $\mathrm{k} 1$ was more than 44.1 diopters in 19 eyes (19\%) of OBL group versus 27 eyes $(27.0 \%)$ in non-OBL eyes.

Table (9): Pre-operative flat keratometry $(\mathrm{k} 1)$ in relation to OBL formation among the studied eyes.

\begin{tabular}{|c|c|c|c|c|c|c|}
\hline \multirow{2}{*}{$\begin{array}{l}\text { Flatkeratometry (k1) } \\
\text { (diopters) }\end{array}$} & \multicolumn{2}{|c|}{$\begin{array}{c}\mathrm{OBL} \\
(\mathrm{N}=100)\end{array}$} & \multicolumn{2}{|c|}{$\begin{array}{l}\text { Non-OBL } \\
(\mathrm{N}=100)\end{array}$} & \multirow{2}{*}{$\begin{array}{c}t- \\
\text { value/ } \\
\chi^{2}\end{array}$} & \multirow{2}{*}{$\begin{array}{c}p- \\
\text { value }\end{array}$} \\
\hline & No. & $\%$ & No. & $\%$ & & \\
\hline $\begin{array}{l}\text { Flat keratometry }(k 1) \\
\text { Mean } \pm \text { SD } \\
\text { Range }\end{array}$ & \multicolumn{2}{|c|}{$\begin{array}{l}42.89 \pm 1.44 \\
(38.8-46.1)\end{array}$} & \multicolumn{2}{|c|}{$\begin{array}{l}43.01 \pm 1.65 \\
(39.1-46.9)\end{array}$} & 0.57 & 0.17 \\
\hline $\begin{array}{c}\text { Flat keratometry }(k 1) \\
\text { Lowest thru } 42 \\
42 \text { thru } 42.9 \\
42.9 \text { thru } 44.1 \\
44.1 \text { thru highest }\end{array}$ & $\begin{array}{l}23 \\
31 \\
27 \\
19\end{array}$ & $\begin{array}{l}23.0 \\
31.0 \\
27.0 \\
19.0\end{array}$ & $\begin{array}{l}28 \\
18 \\
27 \\
27\end{array}$ & $\begin{array}{l}28.0 \\
18.0 \\
27.0 \\
27.0\end{array}$ & 5.3 & 0.15 \\
\hline
\end{tabular}

- Steep keratometry ( $k 2$ ): Regarding preoperative steep keratometry $(\mathrm{k} 2)$ regarding OBL formation among the studied 200 eyes, there was a highly statistically significant difference between both groups ( $p$-value was <0.01).

On distribution of steep keratometry $(\mathrm{k} 2)$, there was no statistically significant difference between OBL and non-OBL ( $p$-value was 0.57 ) where $\mathrm{k} 2$ was more than 45.4 in 21 eyes $(21 \%)$ of OBL group versus 29 eyes $(29.0 \%)$ in non-OBL eyes (Table $10)$.

- Central corneal thickness (um): Regarding preoperative central corneal thickness (um) in relation to OBL formation among the studied 200 eyes, there was statistically significant difference between both groups ( $p$-value was 0.03$)$ being thicker in OBL group (Table 11).

Table (10): Pre-operative steep keratometry (k2) regarding OBL formation among the studied eyes.

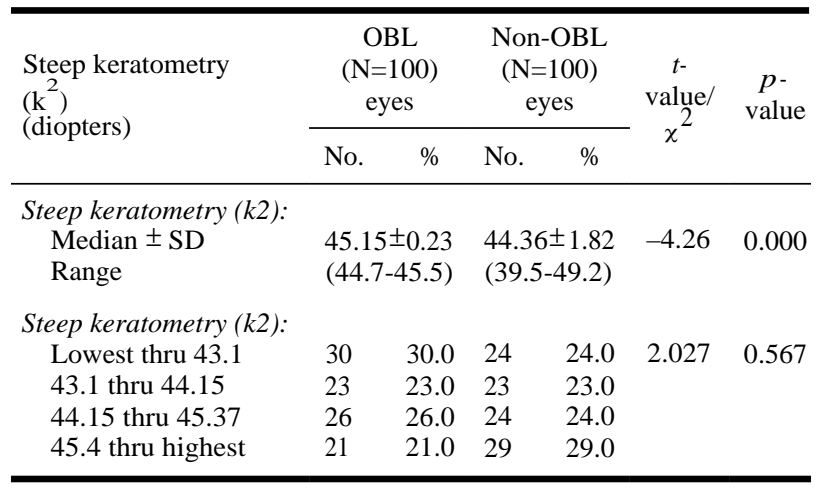

Table (11): Central corneal thickness in relation to OBL formation among the studied eyes.

\begin{tabular}{lllrr}
\hline Pachymetry & \multicolumn{1}{c}{$\begin{array}{c}\text { OBL } \\
(\mathrm{N}=100) \text { eyes }\end{array}$} & $\begin{array}{c}\text { Non-OBL } \\
(\mathrm{N}=100) \text { eyes }\end{array}$ & $\begin{array}{c}\mathrm{Z}- \\
\text { value }\end{array}$ & $\begin{array}{c}p \text { - } \\
\text { value }\end{array}$ \\
\hline Median, IQR & $551.0,44.0$ & $537.5,44.0$ & -2.16 & $0.03^{*}$ \\
Range & $(486.0-600.0)$ & $(484.0-590.0)$ & & \\
\hline
\end{tabular}

Regarding the distribution of central corneal thickness in both groups regarding median of central corneal thickness, there was a statistically significant difference between OBL and non-OBL ( $p$-value was 0.03 ) where the corneal pachymetry was more than $545 \mathrm{um}$ in 57 eyes $(57 \%)$ of OBL group versus 42 eyes $(42.0 \%)$ in non-OBL eyes (Table 12).

Table (12): Distribution of central corneal thickness among the studied groups.

\begin{tabular}{|c|c|c|c|c|c|c|}
\hline \multirow{2}{*}{$\begin{array}{l}\text { Central corneal } \\
\text { thickness (um) }\end{array}$} & \multicolumn{2}{|c|}{$\begin{array}{c}\text { OBL } \\
(\mathrm{N}=100) \text { eyes }\end{array}$} & \multicolumn{2}{|c|}{$\begin{array}{c}\text { Non-OBL } \\
(\mathrm{N}=100) \text { eyes }\end{array}$} & \multirow{2}{*}{$x^{2}$} & \multirow{2}{*}{$\begin{array}{c}p- \\
\text { value }\end{array}$} \\
\hline & No. & $\%$ & No. & $\%$ & & \\
\hline Lowest thru 517 & 22 & 22.0 & 29 & 29.0 & 5.03 & 0.17 \\
\hline 517 thru 545 & 21 & 21.0 & 29 & 29.0 & & \\
\hline 545 thru 563 & 27 & 27.0 & 23 & 23.0 & & \\
\hline 563 thru highest & 30 & 30.0 & 19 & 19.0 & & \\
\hline$<$ median $(545)$ & 43 & 43.0 & 58 & 58.0 & 4.5 & 0.03 \\
\hline$\geq$ median $(545)$ & 57 & 57.0 & 42 & 42.0 & & \\
\hline
\end{tabular}

C- Intraoperative data:

- Corneal flap parameters: There was no statistically significant difference between both groups regarding canal length offset $(\mathrm{mm})$, corneal flap thickness, corneal flap diameter, and flap hinge angle (Tables 13A,13B).

- Canal length offset ( $\mathrm{mm})$ : It begins from the limbus towards flap hinge and in the cross sectional view, begins from the top of conjunctiva towards lamellar cut bed. On comparison between both groups regarding canal length offset $(\mathrm{mm})$, There was a statistically significant difference 
between OBL and non-OBL ( $p$-value was 0.04) where the canal length offset $(\mathrm{mm})$ was more than $0.8 \mathrm{~mm}$ in 28 eyes $(28 \%)$ OBL group versus 12 eyes $(12.0 \%)$ in non-OBL eyes (Table 14$)$.

Table (13A): Pre-operative corneal flap parameters in relation to OBL formation.

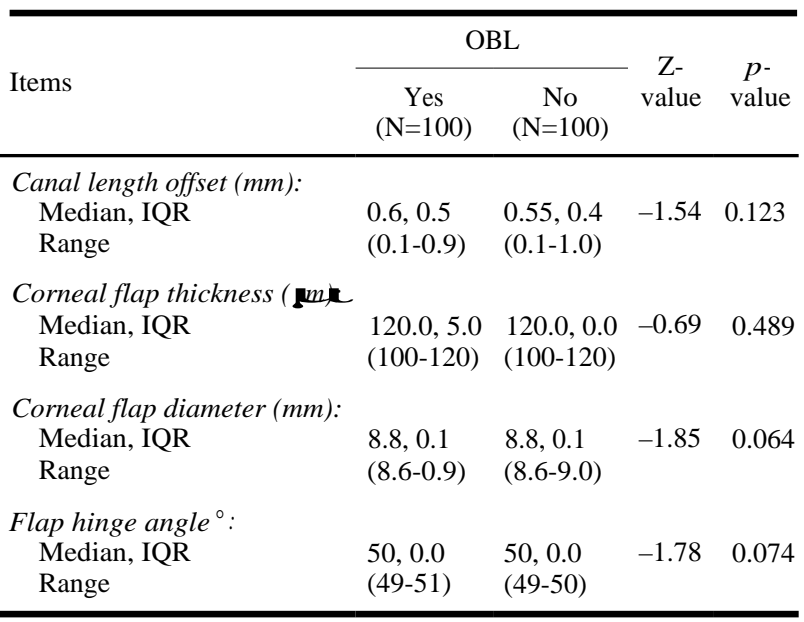

Table (13B): Pre-operative corneal flap thickness and diameter in relation to OBL formation among the studied eyes.

\begin{tabular}{|c|c|c|c|c|c|c|}
\hline \multirow{3}{*}{ Items } & \multicolumn{4}{|c|}{ OBL } & \multirow{3}{*}{$x^{2}$} & \multirow{3}{*}{$\begin{array}{c}p- \\
\text { value }\end{array}$} \\
\hline & \multicolumn{2}{|c|}{ Yes $(N=100)$} & \multicolumn{2}{|c|}{ No $(\mathrm{N}=100)$} & & \\
\hline & No & $\%$ & No & $\%$ & & \\
\hline \multicolumn{7}{|l|}{$\begin{array}{l}\text { Corneal flap } \\
\text { thickness }\end{array}$} \\
\hline$\leq 115$ & 28 & 28.0 & 23 & 23.0 & 0.658 & 0.517 \\
\hline $120(\mathrm{~m})$ & 72 & 72.0 & 77 & 77.0 & & \\
\hline \multicolumn{7}{|l|}{$\begin{array}{l}\text { Corneal flap } \\
\text { diameter }(\mathrm{mm}) \text { : }\end{array}$} \\
\hline $8.7(\mathrm{~mm})$ & 38 & 38.0 & 49 & 49.0 & 2.460 & 0.154 \\
\hline$\geq 8.8(\mathrm{~mm})$ & 62 & 62.0 & 51 & 51.0 & & \\
\hline
\end{tabular}

Table (14): Distribution of canal length ( $\mathrm{mm}$ ) among the studied groups.

\begin{tabular}{|c|c|c|c|c|c|c|}
\hline \multirow{2}{*}{$\begin{array}{l}\text { Canal length } \\
(\mathrm{mm})\end{array}$} & \multicolumn{2}{|c|}{$\begin{array}{c}\mathrm{OBL} \\
(\mathrm{N}=100) \text { eye }\end{array}$} & \multicolumn{2}{|c|}{$\begin{array}{c}\text { Non-OBL } \\
(\mathrm{N}=100) \text { eye }\end{array}$} & \multirow{2}{*}{$x^{2}$} & \multirow{2}{*}{$\begin{array}{c}p- \\
\text { value }\end{array}$} \\
\hline & No. & $\%$ & No. & $\%$ & & \\
\hline Lowest thru 0.4 & 31 & 31.0 & 34 & 34.0 & 8.506 & 0.037 \\
\hline 0.4 thru 0.6 & 24 & 24.0 & 34 & 34.0 & & \\
\hline 0.6 thru 0.8 & 17 & 17.0 & 20 & 20.0 & & \\
\hline 0.8 thru highest & 28 & 28.0 & 12 & 12.0 & & \\
\hline
\end{tabular}

Correlation was done between the development of OBL and each of study variables and revealed no statistically significant differences except weak significant correlation with the central corneal thickness $(r: 0.15, p$-value was 0.03$)$ andalso with $\mathrm{K} 2$, steep meridian ( $r: 0.29, p$-value was $<0.01$ ).

Linear regression analysis: The table below represents the best fitting logistic regression model for OBL formation, the table displays that central corneal thickness, corneal astigmatisms, flat and steep corneal meridianswere statistically significant independent predictor of OBL formation.

Table (15): Logistic regression of OBL depending on presence of risk factors.

\begin{tabular}{|c|c|c|c|c|c|c|}
\hline \multirow[t]{2}{*}{ Variables } & \multirow[t]{2}{*}{ B } & \multirow{2}{*}{$\begin{array}{l}\text { St. } \\
\text { error }\end{array}$} & \multirow[t]{2}{*}{ Sig. } & \multirow[t]{2}{*}{ Beta } & \multicolumn{2}{|c|}{$\begin{array}{c}95 \% \text { C.I. } \\
\text { for } \operatorname{EXP~(B)~}\end{array}$} \\
\hline & & & & & Lower & Upper \\
\hline - Age & 0.009 & 0.008 & 0.262 & 0.11 & -0.006 & 0.023 \\
\hline - Sex & 0.14 & 0.10 & 0.18 & 0.135 & -0.065 & -0.006 \\
\hline - SE & 0.009 & .014 & 0.53 & 0.04 & -0.02 & 0.04 \\
\hline - Refraction & 0.53 & 0.24 & 0.04 & 0.21 & 0.025 & 1.03 \\
\hline - Astigmatism & 0.156 & 0.04 & 0.00 & 0.28 & 0.078 & 0.24 \\
\hline - K1 & -0.141 & 0.03 & 0.00 & -0.43 & -0.197 & -0.08 \\
\hline - $\mathrm{K} 2$ & 0.262 & 0.03 & 0.00 & 0.71 & 0.198 & 0.32 \\
\hline $\begin{array}{l}\text { - Central corneal } \\
\text { thickness }\end{array}$ & 0.004 & 0.001 & 0.00 & 0.24 & 0.002 & 0.007 \\
\hline - Corneal flap thickness & -0.008 & .006 & 0.19 & -0.08 & -0.02 & 0.004 \\
\hline - Corneal flap diameter & 0.929 & .538 & 0.09 & 0.13 & -0.13 & 1.98 \\
\hline - Flap hinge angle & 0.191 & .162 & 0.24 & 0.09 & -0.13 & 0.51 \\
\hline - Canal length offset & 0.183 & .112 & 0.22 & 0.07 & -0.08 & 0.36 \\
\hline
\end{tabular}

\section{Discussion}

Femtosecond-Laser (FSL) becomes one of the most important advances in refractive surgeries as it increases the predictability, precision and accuracy of LASIK flap creation. Also, it allows for flap customization (thickness, diameter, side cut angle) and is better for thin corneas [4]

OBL is one of the most common complications of FSL. This trapped gas may create difficulty in lifting the flap, limit a patient's ability to fixate properly during ablation and it also may interfere with pupil tracker during excimer laser ablation if it obscures all or part of the pupil [6]

The main goal of this study was to determine risk factors responsible for OBL formation in FSLassisted LASIK. This retrospective study was conducted on 200 eyes of 101 patients who were eligible for FSL-assisted LASIK using FS200. One hundred eyes had OBL and 100 eyes did not develop OBL.

In the present study, thick cornea, steep keratometry and increased corneal canal length were risk factors for OBL formation during surgeries. Regarding linear regression analysis, CCT and increased corneal astigmatism were statistically significant independent predictor of OBL formation.

As thicker corneas can provide greater rigidity and produce more resistance, steeper corneas could be affected by greater pressure that leads to escape of gas bubbles intrastromal. 
Lower astigmatism leads to applanation pressure that is more radially uniform so there is no meridian that allows for easier outflow of the gas bubbles to the conjunctival space versus high astigmatism where there is a meridian in which the applanation pressure is relatively lower. This was stated by wu et al., [10] . Further studies are needed to evaluate the magnitude and direction of astigmatism as a risk factor for OBL formation.

Courtin et al., [5] in a retrospective study on 198 eyes, had FSL-assisted LASIK performed by WaveLight FS200 laser, found correlation between CCT and OBL. As thicker corneas were associated with more frequent and large OBL, the present result study was comparable with that study regarding correlation between thicker cornea and OBL formation.

Liu et al., [7] assessed the incidence, risk factors, and impact on visual outcomes of OBL produced by interlase FSL $(60 \mathrm{kHz})$ during LASIK. They stated that OBLs tend to occur in thicker corneas even with a $60 \mathrm{kHz}$ FSL. They hypothesized that the occurrence of an OBL may be affected by the biomechanical properties of the corneaIt has been suggested that corneal thickness is positively correlated with corneal hysteresis and the corneal resistance factor. Thicker corneas can provide greater rigidity and produce more resistance.

In the present study, thicker corneas tended to produce OBLs, even with a $200 \mathrm{kHz}$ FSL.

Kaiserman et al., [9] studied the incidence, characteristic, risk factors and sequels of OBL created by the interlase $(15 \mathrm{kHz})$. The study conducted on 149 eyes which had LASIK for myopic astigmatism. Forty eight eyes $(56.4 \%)$ developed OBL. They found that a smaller flap and thicker cornea were associated with a higher incidence of OBL using a $15 \mathrm{kHz}$ FSL for flap creation. They discussed that if flap diameter increased, being the $\mathrm{S}$ contact glass fixed diameter $8.5 \mathrm{~mm}$, the distance between the flap edge and the contact glass margin would reduce, ensuring a smoother emission of the compressed air generated by the intracorneal FSL action as a relief valve differently, a smaller flap does not allow a "safety valve" phenomenon, and would facilitate the gas entrapment into stromal lamellae and the formation of OBL.

This as in agreement with the results of Matropasqua et al., 2017 who conducted that as a significant reduction and extension of OBL incidence were evident when LASIK flap settings diameter was increased, and flap edge was closer to contact glass border. They use Visumax Carl Zeiss (500
$\mathrm{kHz}$ ) FSL, planned with different flap diameters $(7.9,8.0,8.2 \mathrm{~mm})$ and the same laser energy and power settings.

In the present study, a wider flap diameter than the contact glass ranged from $8.6 \mathrm{~mm}$ to $9.0 \mathrm{~mm}$ was used and there were no significant correlation between OBL formation and different flap diameters.

Jung et al., [8] conducted a study to determine risk factors of OBL formation on 41 eyes. They suggested that steep corneal curvature, a thick cornea, and a hard-docking technique could be risk factors for OBL occurrence as steeper corneas could be affected by greater pressure when the patient interface touches the cornea. Thicker corneas could provide greater rigidity and more resistance, so an OBL could occur more frequently.

This was comparable with the present study in correlation between thicker and steeper corneas and OBL formation.

Wu et al., [10] in a retrospective study on 198 eyes, had FSL-assisted LASIK. OBL happened in 118 eyes. They found that less astigmatic, thicker and smaller corneas increased the risk of OBL using the original technique for flap creation. This was comparable with the present study in correlation between steeper and thicker corneas and OBL formation.

Wei et al., [11] had a case control study on 60 consecutive patients (120 eyes) with unilateral OBL during FS-LASIK. Eyes were divided into OBL (the OBL eyes) and OBL-free groups (the fellow eyes) based on the occurrence of OBL. The OBL eyes were considered as case group while the fellow eyes were considered as paired control group. The main difference between the fellow eyes of one patient was the presetting canal length offset value when the outlet was located at the posterior border of the corneoscleral limbus, the incidence of OBL was significantly low. Corneal astigmatism is also an independent protective factor for OBL formation. There was no significant correlation between $\mathrm{OBL}$ formation and refraction.

The corneal curvature was steeper than the curvature of sclera. When the outlet was located at cornea, the canal undertook more large pressure and had lower probability of patency. So, OBL was easy to happen. The FSL cannot penetrate the sclera due to its opaque characteristic. When the gas diffusing canal outlet was located at sclera, the outlet of gas diffusing canal did not open and the canal could not communicate with the atmos- 
phere. Thus, the gas could not diffuse and led to OBL.

In the present study there was correlation between OBL formation and thick corneas, increase of corneal astigmatism and increase corneal canal length offset.

Mastropasqua et al., [1] studied 108 patients (216 eyes) with bilateral hyperopia, OBL was formed in 35 eyes $(6.1 \%)$. They found that steeper cornea and a hard-docking technique could be risk factors. Also, laser energy and pulse rate were determinants in flap creation by a FSL and in OBL incidence.

This was comparable with the present study in correlation between steeper corneas and OBL formation and incomparable with the present study regarding refraction. As 6 eyes were hyperopic, only 2 eyes ( $1 \%$ of total of the studied eyes) had developed OBL and 194 were myopic, 98 eyes of them (49\% of the studied eyes) had developed OBL. There was no correlation between OBL formation and refraction.

\section{Conclusion:}

OBL is one of FSL complications. Increased corneal thickness, corneal canal length offset and steep keratometry were risk factors of OBL formation during surgeries. Regarding regression analysis; central corneal thickness and corneal astigmatism were statistically significant independent predictor of OBL formation.

\section{References}

1- MASTROPASQUA L., CALIENNO R., LANZINI M., SALGARI N., VECCHI S.D., MASTROPASQUA R. and NUBILE M.: Opaque bubble layer incidence in Femtosecond laser-assisted LASIK: Comparison among different flap design parameters. Int. Ophthalmol., (37): 635-41, 2017.
2- CHEN X., WANG Y., ZHANG J., YANG S. and ZHANG L.: Comparison of ocular higher-order aberrations after SMILE and wavefront-guided femtosecond LASIK for myopia. BMC Ophthalmol., (1) 42-52, 2017.

3- KASETSUWAN N., SATITPITAKUL V., PUANGSRICHARERN V., REINPRAYOON U. and PARIYAKANOK L.: Comparison of performances of femtosecond laser and microkeratome for thin-flap laser in situ keratomileusis. Lasers Surg. Med., 48 (6): 596-601, 2016.

4- ARISTEIDOU A., TANIGUCHI E.V., TSATSOS M., MULLER R., MCALINDEN C., PINEDA R. and PASCHALIS E.I.: The evolution of corneal and refractive surgery with the femtosecond laser. J. Eye Vis. (Lond), (2): 2-12, 2015.

5- COURTIN R., SAAD A., GUILBERT E., GRISE-DULAC A. and GATINEL D.: Opaque bubble layer risk factors in Femtosecond laser-assisted LASIK. J. Refract. Surg., 31 (9): 608-12, 2015

6- KANELLOPOULOS A.J. and ASIMELLIS G.: Longterm bladeless LASIK outcomes with the FS200 Femtosecond and EX500 Excimer Laser workstation: The Refractive Suite. Clin. Ophthalmol., 7: 261-9, 2013.

7- LIU C.H., SUN C.C., HUI-KANG MA D., CHIEN-CHIEH HUANG J., LIU C.F., CHEN H.F. and HSIAO C.H.: Opaque bubble layer: Incidence, risk factors, and clinical relevance. J. Cataract. Refract. Surg., 40 (3): 435-40, 2014.

8- JUNG H.G., KIM G. and LIM T.H.: Possible risk factors and clinical effects of an opaque bubble layer created with femtosecond laser-assisted laser in situ keratomileusis. J. Cataract. Refract. Surg., 41: 1393-9, 2015.

9- KAISERMAN I., MARESKY H.S., BAHAR I. and ROOTMAN D.S.: Incidence, possible risk factors, and potential effects of an opaque bubble layer created by a femtosecond laser. J. Cataract. Refract. Surg., 34: 41723, 2008.

10- WU N., CHRISTENBURY J.G., DISHLER J.G., BOZKURT T.K., DUEL D., ZHANG L. and HAMILTON D.R.: A technique to reduce incidence of oaque bubble layer formation during LASIK flap creation using the VisuMax Femtosecond laser. J. Refract. Surg., 33 (9): 584-90, 2017.

11- WEI C.H., DAI Q.Y., MEI L.X., GE Y., ZHANG P.F. and SONG E.: Paired eye-control study of unilateral opaque bubble layer in femtosecond laser assisted laser in situ keratomileusis. Int. J. Ophthalmol., 12 (4): 654-9, 2019. 


\section{دراسة عوامل الخطر لحدوث الطبقة الفقاعية المعتمة

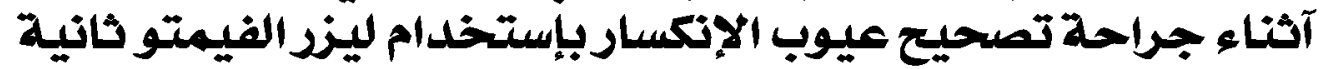

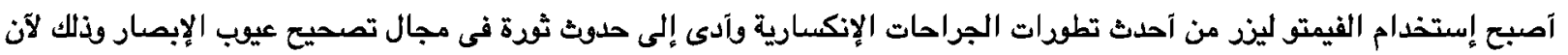

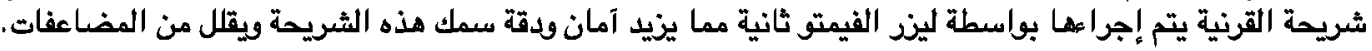

يوجد بعض المضاعفات الخاصة بالفيمتوليزر مثل تكفن فقاعات الغاز والتى قد تسبب صعوبية فى رفع شريحة القرنية آثناء إجراء الجراء الجراحة، حيث آن الغاز لا يق فى مستوى واحد داخل القرنية، كما يعوق البرمجيات المحددة من تبع قزحية عين المريض فئ آثناء عملية تصحيح الإبصاء.

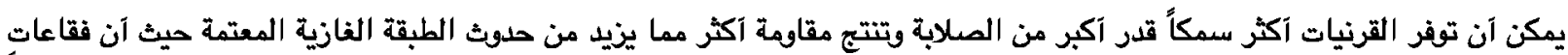

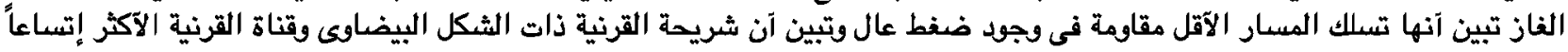
تميل إلى تقليل نسب حدوث هذه الطبقة الفازية المعتمة.

الهدف من إجراء البحتث: دراسة عوامل الخطر المسيبة الطبقة الفقاعية المعتمة فى بعض الحالات التى حدثت بها آثناء جراحة تصحيح

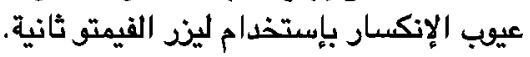

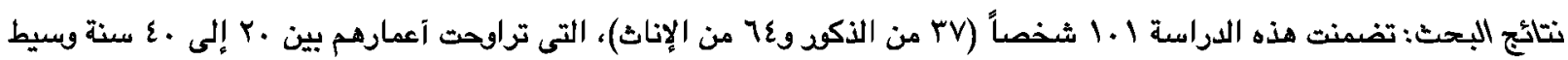
الآعمار •ب سنة.

تم جدولة النتائج وتحليلها إحصائياً. لم يكن هناك فروق ذات دلالة إحصائية فيما يتعلق بالمكافئ الكرعى وقصر النظر آو طول النظر آو الإستجماتيزم.

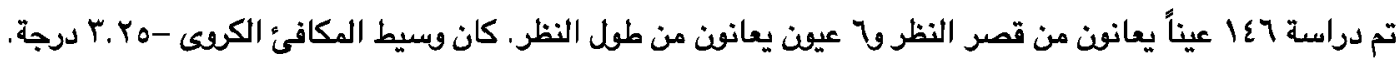

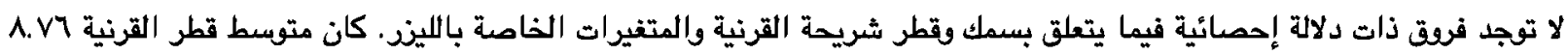

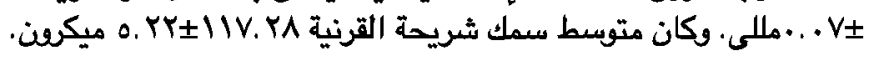

كان هناك فروق ذات دلالة إحصائية فيما يتعلق بسمك القرنية وطول قناة القرنية والإستجماتيزم ودرجة تحدب القرنية القرنية. كان وسيط القرنية

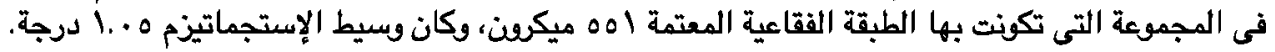
النتائج الموجودة فى دراستتا تشير إلى آن زيادة سمك القرنية وطول قناة القرنية والإستجماتيزم ودرجة تحدب القرنية عوامل مسبية لحلوث الطبقة الفقاعية المعتمة.

الإستتاج النهائى: زيادة سمك القرنية وطول قناة القرنية والإستجماتيزم ودرجة تحدب القرنية من العوامل المسيبة لحدوث الطبقة الفقاعية

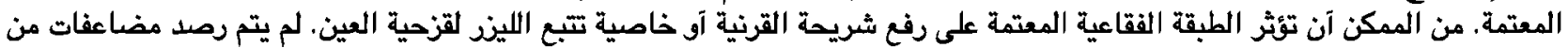
حدوث الطبقة الفقاعية المعتمة آثناء الجراحة. 